\title{
Editorial
}

\section{Other men's (and women's) flowers}

The front page of an anthology of poems collected by Lord Wavell carries a charming quotation from Montaigne: 'I have gathered a posie of other men's flowers and nothing but the thread that binds them is my own'. In scientific journals we collect research papers, rather than flowers, but there is increasing pressure on researchers, and editors, to ensure that the ownership of the research is correctly ascribed. Ideally, research will be promptly and clearly reported in an appropriate journal and correctly attributed to the people who did the research. We hope that this is usually what actually happens.

However, sometimes mistakes are made. In this issue we, the editors of the International Journal of Obesity and the European Journal of Clinical Nutrition, have the unpleasant task of retracting a paper which was, without the knowledge of the editors, submitted to, and published by both journals. Some may ask why multiple publication is a crime: if the data are correct and interesting, why not present them to as large a public as possible?

There are several answers to this question. From the viewpoint of the reader who tries to keep abreast of the literature in the field, multiple publication means that more journals have to be scanned to collect the same amount of information. For the editors and reviewers, time is wasted assessing and improving a paper which is meanwhile having the same work done by other people for another journal. Those who maintain bibliographic databases, or attempt meta-analyses in the field, are confused: does the second paper refer to the same series of people with a given condition or a different one? Those who use citation indices as a measure of the research output of an individual or an institution are confused: do these two publications represent two research programmes, or merely two descriptions of the same research programme? For all of these reasons, contributors to this journal are asked to sign a letter confirming that they have not submitted the paper elsewhere, and will not do so while it is under consideration by this journal. To dissuade authors from such misconduct, COPE (the Committee on Publication Ethics) suggests that authors who deliberately violate this condition should be barred from publication in the journals concerned for a period of two years.

Other problems are more difficult to resolve. For example, an excellent review of the epidemiological evidence on the association between dietary fat and obesity was published in the European Journal of Clinical Nutrition in 1995. Two years later, in a supple- ment of the International Journal of Obesity, a review was published on a similar topic. In the opinion of an independent referee, who was asked to examine the second paper for possible plagiarism, a substantial part of the text and associated figures were deemed to be remarkably similar (see report following this Editorial). Of course, two reviews about the same topic will draw on the same pool of evidence, so overlapping references are to be expected, just as two people gathering flowers from the same area will make similar collections. However, it would be expected that the way in which data were arranged and interpreted would differ, so the second reviewer could truthfully claim 'the thread that binds them is my own'.

So how can a conscientious worker escape a charge of plagiarism if he reviews an area which has previously been reviewed? The answer, if the previous review was comprehensive, and he has no new insight to offer, is that the field is not yet suitable for another review. If parts of the field have already been well covered, but other parts have not, then it is up to the latest reviewer to cite and summarise the previous review as concisely as possible, and move on to the part where he has new data, or new interpretations to suggest.

Systematic reviews and meta-analyses present a particularly difficult problem concerning citation and ownership of data. A good meta-analysis may be a superb synthesis of current knowledge in a field, and if so it will be more frequently cited than any individual study in the field. But the meta-analyst may not have contributed a single datum point by his own experimental work, but relied entirely on the published (or unpublished) work of other people, so it seems unjust that all the credit should go to the florist who presents a magnificent bouquet, and none to the gardeners who grew the flowers.

The volume of bio-medical literature is now so great that it threatens to overwhelm the serious scholar in any but the smallest specialist field. Publications are not only a means of sharing findings with fellowscientists, but are also the currency with which academic promotion and funding is purchased. It is the responsibility of authors of papers to ensure that they give proper credit to other investigators upon whose published work they have built their own research: failure to make such proper citations is, in effect, stealing the credit for the work of others, by implying that it is their own work. Duplicate publication or plagiarism may be detected by search of electronic databases, or by attentive readers who notify the editors of suspected abuses. Researchers, reviewers, 
readers and editors must work together to see that a high standard of publication ethics is maintained. We would be interested to hear your views on this topic, in the form of a letter suitable for publication.
Letters for publication in IJO or EJCN should be sent to MJS or JSG, respectively. The report of the independent referee referred to above,

is printed below.

J-P Després

IA Macdonald

JS Garrow

MJ Stock

International Journal of Obesity

JC Seidell

European Journal of Clinical Nutrition

\section{Report on Reviews by Lissner and Heitmann (EJCN 1995; 49: 79-90) and Golay and Bobbioni (IJO 1997; 21 (Suppl 3): S2-S11)}

I have been asked to compare these two articles on dietary fat and obesity, as the question has been raised whether the paper of Golay and Bobbioni could possibly be regarded as an example of plagiarism.

I have scrutinised the two papers and have the following comments:

In the Golay paper, a reference is clearly made to the paper by Drs Lissner and Heitmann. Some of the figures, as well as Table 1, are based on similar background material.

Starting with the reference list of the Golay paper, about $60 \%$ of the references are identical. The references in the first part of the papers are almost identical in order. Although the Golay paper is published two years later than the Lissner review, the only papers in the Golay review that appear with a date later than 1995 are the Lissner-Heitmann study and a paper from Golay's own group published in 1996.

The Golay paper consists of two parts. The first one is an epidemiological analysis of the relationship between fat consumption and obesity. The second part of the paper concerns mechanisms by which a high fat diet might stimulate hyperphagia, a topic which is only briefly addressed in the Lissner/ Heitmann paper. Here the two reviews clearly are different and the references used by Golay/Bobbioni in this section are not similar to the Lissner/Heitmann reference list.

Thus the question of plagiarism concerns the first part of the Golay/Bobbioni paper, where clear similarities can be observed, the structure in thought is almost identical, the references are almost identical and even the subtitles are more or less identical.

Some sections contain a wording in the Golay/Bobbioni paper which is practically identical to the Lissner/Heitmann study. Examples:

Golay/Bobbioni p. S3, last sentence under section 'Secular trends' is identical to the text on bottom of p. 81 in Lissner/Heitmann.

The reference in Golay/Bobbioni on p. S4 to the work of Tremblay et al (refs 11, 12) contains almost the identical wording as in Lissner/Heitmann, bottom of p. 84 .

Reference 24 to Rissanen likewise on p. S4 is almost identical to the lower part, right column, of p. 84 .

In the second part of the Swiss study, the similarities disappear and the conclusions reached in the Golay/Bobbioni study, concern matters other than these in the review by Lissner/Heitmann, who concentrate more on the epidemiological approach. The section by Lissner-Heitmann with the subtitle 'Consistency with experimental data' contains several references which can be found in the Golay/Bobbioni paper, but here the Swiss group has clearly developed their own thoughts and have detailed information.

In summary, this comparison has revealed that the first part of the Golay/Bobbioni paper in concept, design and often also wording, is strikingly similar to the Lissner/Heitmann review. To me it is clear that Golay and Bobbioni have relied heavily on the Lissner/Heitmann material in the first part of the study, where subtitles, figures, tables, references and the flow of thought is very similar. The second part of both papers goes in different directions and the conclusion, as well as the summaries, do not overlap.

S Rössner, MD, PhD

Professor of Health Behaviour Research, Director, Obesity Unit, Huddinge University Hospital, 